\title{
Combined Right Ventricular Outflow Tract Epicardial and Endocardial Late Potential Ablation for Treatment of Brugada Storm: A Case Report and Review of the Literature
}

Sandeep A. Saha · Kousik Krishnan · Christopher Madias · Richard G. Trohman

Received: June 1, 2016/ Published online: September 19, 2016

(C) The Author(s) 2016. This article is published with open access at Springerlink.com

\section{ABSTRACT}

A 34-year-old man with Brugada syndrome (BrS) presented with electrical storm, manifested as multiple appropriate shocks from his implantable cardioverter-defibrillator over a period of 7 hours. He had not tolerated prior treatment with quinidine, and had self-discontinued cilostazol citing persistent palpitations. After stabilization with intravenous isoproterenol, an electrophysiology study was performed but no spontaneous or induced ventricular ectopic beats were identified. A three-dimensional (3D) endocardial electro-anatomic map of the right ventricular outflow tract (RVOT), pulmonic valve, and pulmonary artery, as well as a 3D epicardial map of the RVOT, were created. Low voltage, complex, fractionated electrograms and late potentials were targeted

Enhanced content To view enhanced content for this article go to http://www.medengine.com/Redeem/ BDB5F0601B2CC15A.

S. A. Saha $(\varangle) \cdot$ K. Krishnan · C. Madias .

R. G. Trohman

Section of Cardiac Electrophysiology, Division of

Cardiology, Department of Medicine, Rush

University Medical Center, Chicago, IL, USA

e-mail: sandeep_a_saha@rush.edu for irrigated radiofrequency ablation both endocardially and epicardially. Post-procedure, he was maintained on cilostazol (referring clinician preference), and has had no further ventricular tachyarrhythmia episodes over the past forty-one months. We propose that this novel ablation strategy may be useful for acute management of selected patients with BrS.

Keywords: Brugada syndrome; Implantable cardioverter-defibrillator; Late potentials; Radiofrequency ablation

\section{INTRODUCTION}

Brugada syndrome (BrS) is characterized by the presence of characteristic electrocardiographic (ECG) changes (coved ST-segment elevation $\geq 2 \mathrm{~mm}$ with a right bundle-branch pattern) in the right-sided precordial leads (leads $\mathrm{V}_{1}$ through $V_{3}$ ), either at baseline, or after administration of a class I (sodium channel blocking) antiarrhythmic drug associated with cardiac arrest or sudden death in the absence of demonstrable structural heart disease [1]. Clinical characteristics include spontaneous or 
induced ventricular tachycardia (VT) or ventricular fibrillation (VF), atrial fibrillation, a family history of sudden death at age $<45$ years, similar ECG abnormalities in family members, syncope, or nocturnal agonal respiration [2]. The only proven therapeutic option for prevention of sudden death in BrS patients is the implantable cardioverter-defibrillator (ICD) [2], although recent data have also demonstrated the efficacy of anti-arrhythmic agents (mostly quinidine) that block the transient outward potassium current $\left(\mathrm{I}_{\mathrm{to}}\right)$ in patients who can tolerate these medications long-term [3]. Electrical storm in BrS patients (Brugada storm) is a rare but devastating clinical entity. Acute management of this condition usually includes medical therapy directed at augmenting the slow inward calcium current $\left(\mathrm{I}_{\mathrm{CaL}}\right)$ (intravenous isoproterenol), and possibly counter-acting the effects of $\mathrm{I}_{\text {to }}$ (quinidine and disopyramide) [2, 4].

Ablation is rarely performed for Brugada storm. Current practice guidelines give ablation a class IIb recommendation for treatment of patients with Brugada storm [2]. Prior studies proposed the use of endocardial ablation of focal premature ventricular complexes (PVCs) within the right ventricular outflow tract (RVOT) or the right-sided His-Purkinje system as a means of preventing VT/VF episodes in BrS patients [5]. Subsequent animal studies suggested that radiofrequency (RF) ablation of the epicardial surface of the RVOT may be more effective in eliminating VT in BrS [6]. RF ablation of epicardial ventricular foci may prevent future VT/VF episodes in BrS patients [7]. Newer insights into the pathophysiology of ventricular arrhythmias in BrS has led to use of other ablation targets such as late potentials, as well as low-voltage, fractionated electrograms on voltage mapping [8]. In this paper we describe a novel combined (endocardial and epicardial) ablation in the RVOT performed in a young man with Brugada storm.

\section{CASE}

Informed consent was obtained from the patient for being included in this report. A 34-year-old male with a history of BrS with a dual-chamber ICD and six prior shocks for documented VF was admitted to our hospital after three appropriate shocks within 7 hours. His initial diagnosis of BrS was based on the presence of Brugada pattern on surface ECG and recurrent episodes of nocturnal (non-exertional) syncope. An electrophysiology (EP) study revealed a prolonged $\mathrm{H}-\mathrm{V}$ interval of $75 \mathrm{~ms}$, and a dual-chamber ICD was implanted. At initial presentation, cardiac magnetic resonance imaging did not reveal any structural heart disease or late gadolinium enhancement, and diagnostic coronary angiography revealed normal coronary anatomy and absence of luminal stenosis. He continued to have occasional episodes of palpitations but did not have any shocks for the first five years post ICD implant. After receiving four appropriate shocks (all for VF episodes while asleep) over a period of two years, he was admitted and started on extended-release quinidine gluconate $324 \mathrm{mg}$ every 8 hours, but this medication was stopped due to QTc prolongation (from 430 to $535 \mathrm{~ms}$ after the 3rd dose), dizziness, and gastrointestinal distress. He suffered two more VF shocks from his ICD about ten months later, and was admitted and started on cilostazol $100 \mathrm{mg}$ twice daily. However, after two months of therapy, the patient stopped taking the cilostazol citing persistence of palpitations. 
After being admitted to the coronary intensive care unit (CICU), he received another VF shock, and IV isoproterenol (titrated to a sinus rate of 120 beats per minute) was started overnight. Interrogation of his device showed that all of his VF episodes were triggered by premature ventricular complexes with short coupling intervals (Fig. 1). After ruling out other causal factors including fever, electrolyte abnormalities, and myocardial ischemia, the patient was offered the option of either restarting medical therapy or ablation. Given the acute presentation of
Brugada storm, an ablative strategy was chosen to prevent further shocks. An EP study was performed, but no PVCs were present at baseline, and no PVCs or VT could be induced with burst pacing or programmed stimulation. A three-dimensional (3-D) map of the RVOT, pulmonic valve, and pulmonary artery was constructed using an EnSite ${ }^{\mathrm{TM}} \mathrm{NavX}^{\mathrm{TM}}$ (Endocardial Solutions, St Jude Medical, Minneapolis, MN, USA) system. After a coronary angiogram revealed normal coronary arteries, percutaneous epicardial access was obtained, and a 3-D epicardial map of the

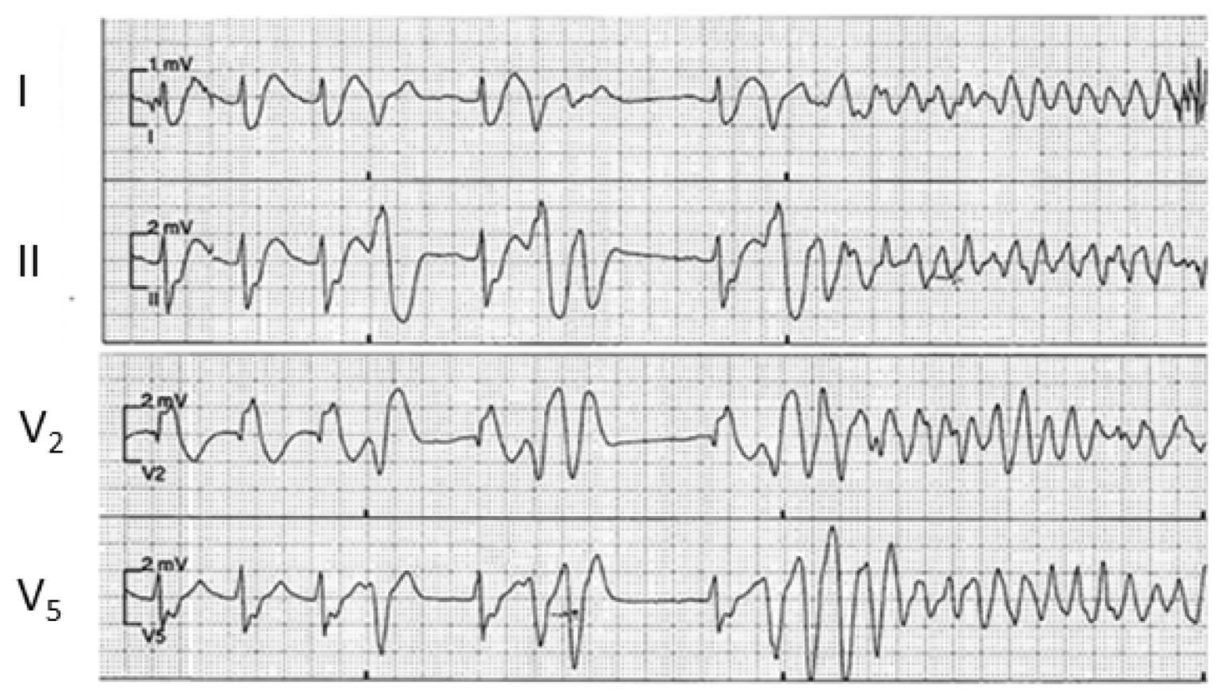

\section{A}

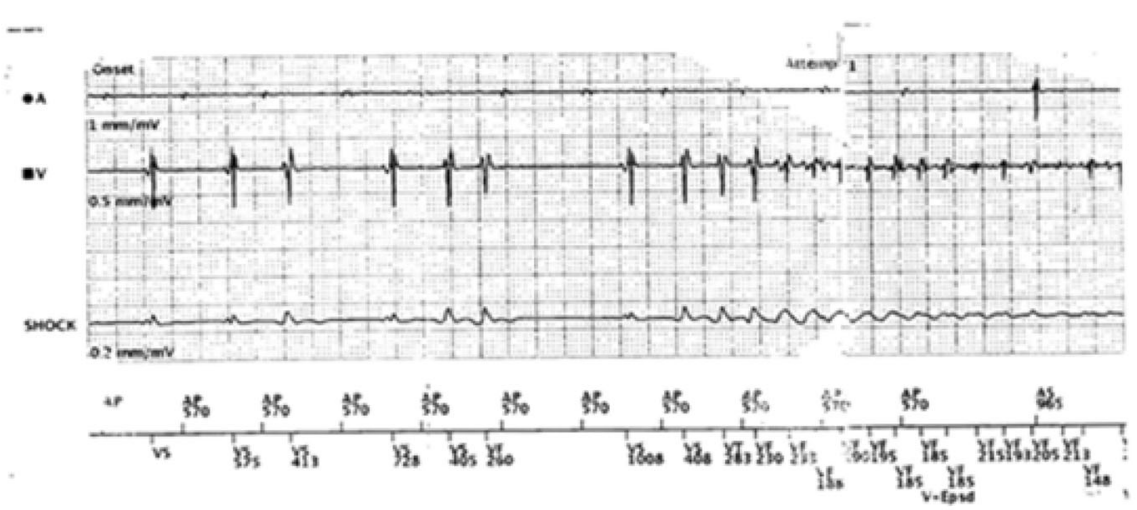

B

Fig. 1 Initiation of ventricular fibrillation in our patient. Top panel a Surface electrocardiogram (ECG) showing premature ventricular complexes likely arising from the right ventricular outflow tract. Bottom panel b Intracardiac electrogram from implantable cardioverter-defibrillator interrogation of the same event 
RVOT and LVOT was also created using the EnSite NavX system. Low voltage, complex, fractionated electrograms and late potentials in the RVOT were identified up to $185 \mathrm{~ms}$ after the QRS complex on the endocardial surface (Fig. 2), and up to $246 \mathrm{~ms}$ after the QRS complex on the epicardial surface (Fig. 3) on 3-D electro-anatomic mapping. These areas were targeted for RF ablation using a $3.5 \mathrm{~mm}$ irrigated tip ablation catheter (Thermocool $^{\circledR}$ SF, Biosense Webster Inc., South Diamond Bar, CA, USA) (Figs. 4, 5a-b). A total of 107 ablation lesions were delivered, of which twenty-eight ablation lesions were delivered on the endocardial surface, and seventy-nine ablation lesions were delivered on the epicardial surface of the RVOT. Towards the end of the procedure, we noted widening of the QRS duration and increased ST elevation. Emergent blood tests revealed hyperkalemia with a serum $\mathrm{K}^{+}$level of
$8.2 \mathrm{mEq} / \mathrm{l}$, serum bicarbonate of $18 \mathrm{mEq} / \mathrm{l}$, serum lactic acid of $3.3 \mathrm{mmol} / \mathrm{l}$, a serum $\mathrm{pH}$ of 7.32 , elevated serum creatinine of $1.35 \mathrm{mg} / \mathrm{dl}$, and creatine kinase of $1220 \mathrm{U} / \mathrm{ml}$. Review of the anesthesiologist's records revealed that the patient was receiving sevoflurane during the case, with intermittent use of intravenous ephedrine. Aggressive treatment with intravenous $50 \%$ dextrose followed by IV insulin, IV calcium chloride, IV sodium bicarbonate, and oral sodium polystyrene sulfonate were given, and the ECG abnormalities resolved. The patient was monitored in the CICU after the procedure, and did not have any recurrence of hyperkalemia or ventricular tachyarrhythmias. He was discharged on day five post-procedure after being restarted on oral cilostazol $100 \mathrm{mg}$ twice daily as per referring physician preference. He was briefly admitted two weeks after the

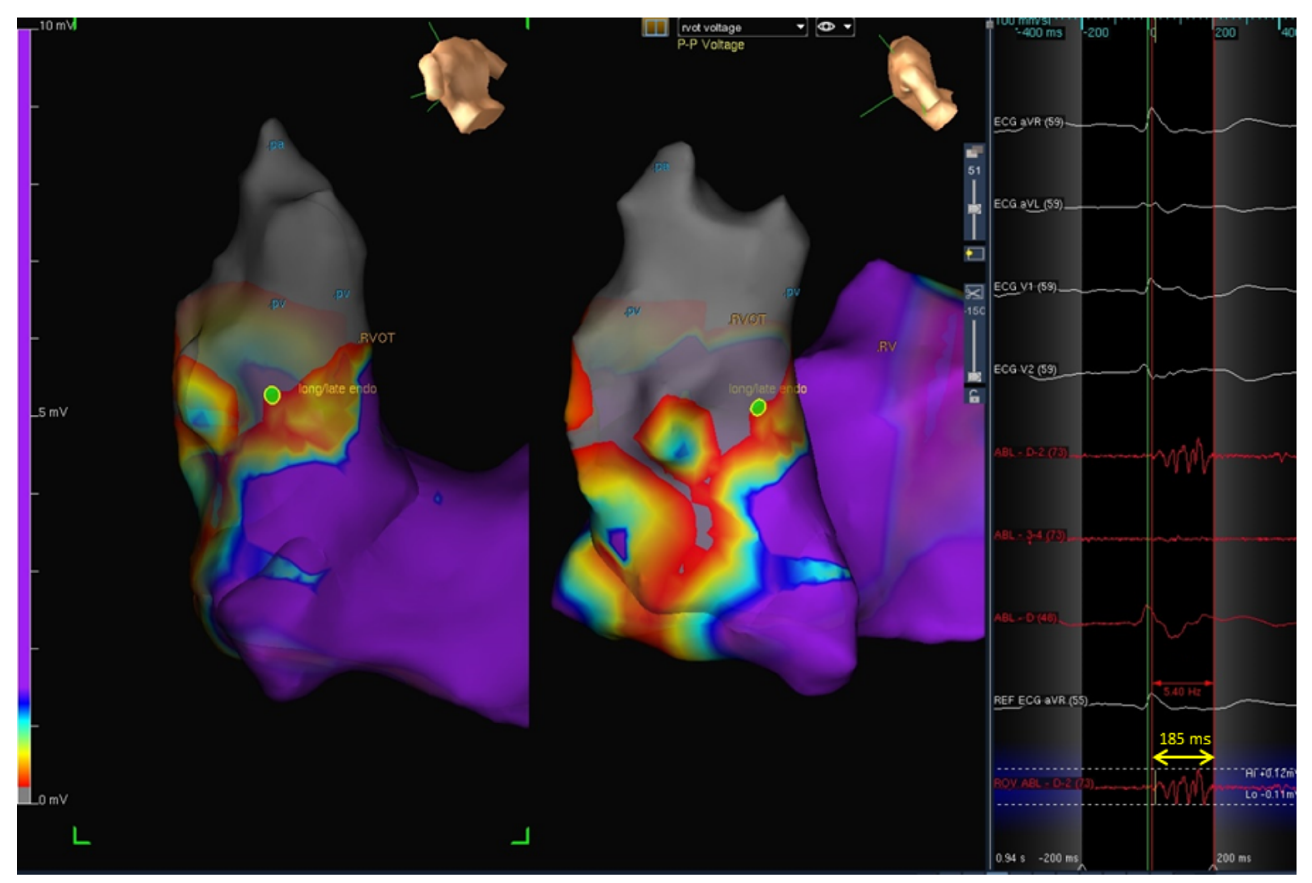

Fig. 2 3-D voltage map showing late fractionated potentials in the right ventricular outflow tract (RVOT) endocardium. Note the unipolar electrogram at the selected site (green dot) showing a delayed fractionated signal which extends $185 \mathrm{~ms}(\mathrm{~ms})$ beyond the R wave (yellow arrowbeads) 


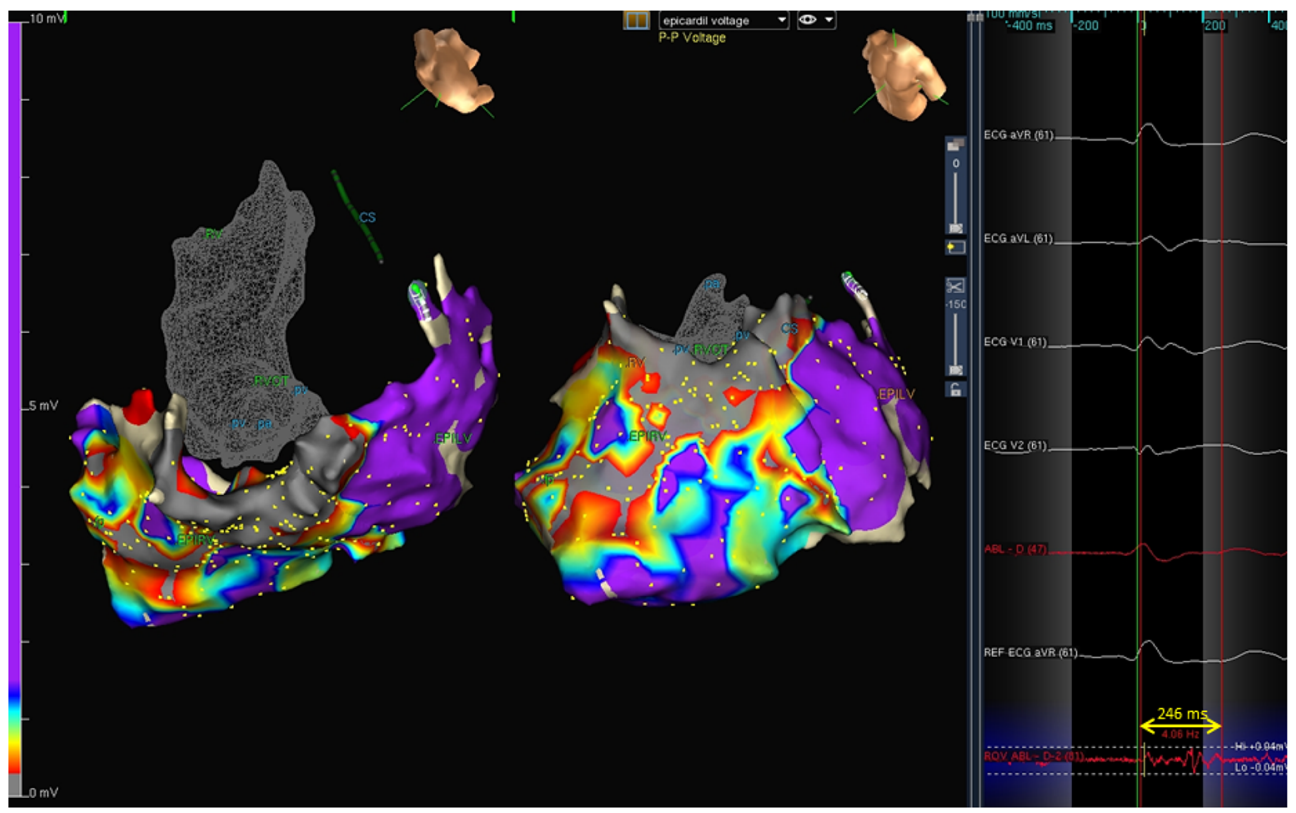

Fig. 3 3-D voltage map of the right ventricular utflow tract (RVOT) and anterior RV free wall showing late fractionated potentials in the RV epicardium. Note the unipolar electrogram showing a delayed fractionated signal which extends $246 \mathrm{~ms}$ ( $\mathrm{ms}$ ) beyond the Q wave (yellow

procedure with pericarditis which resolved after a one-week course of ibuprofen.

In view of his clinical presentation of $\mathrm{BrS}$ and the occurrence of unexpected hyperkalemia and metabolic acidosis with the use of sevoflurane, genetic testing for BrS-related mutations as well as latent neuromuscular disorders was offered to the patient, but he declined.

Close monitoring of his clinical status and interrogation of his ICD over a period of forty-one months post-ablation have revealed no further episodes of VT/VF.

\section{DISCUSSION}

$\mathrm{BrS}$ is an autosomal dominant genetic disorder with incomplete penetrance, but the precise genetic basis of this condition is known in only $\sim 30 \%$ of the affected individuals [9]. To date, mutations in 19 different genetic loci arrowheads). Note that the low-voltage signals are localized over the anterior wall of the RV (labeled EPIRV) and right ventricular outflow tract, while the mapped portion of the left ventricular epicardium (labeled EPILV) shows a normal voltage map (purple)

have been associated with $\mathrm{BrS}$, which either cause loss of function of the sodium channels or the L-type calcium channels, or cause a gain in function of the potassium channels. Mutations in the SCN5A gene account for $\sim 15 \%$ of $\mathrm{BrS}$ patients, leading to either loss of expression or altered function of the $\alpha$-subunit of the cardiac sodium channel [9]. The majority of symptomatic BrS patients are male, and male gender is associated with a sevenfold higher risk for cardiac arrest in patients with BrS [10].

Although ICDs are the mainstay for prevention of sudden arrhythmic death in patients with $\mathrm{BrS}$, they do not reduce the frequency of malignant ventricular arrhythmias. Multiple ICD shocks, albeit appropriate, can be psychologically devastating to the patient $[11,12]$, may cause acute myocardial injury [13], myocardial 


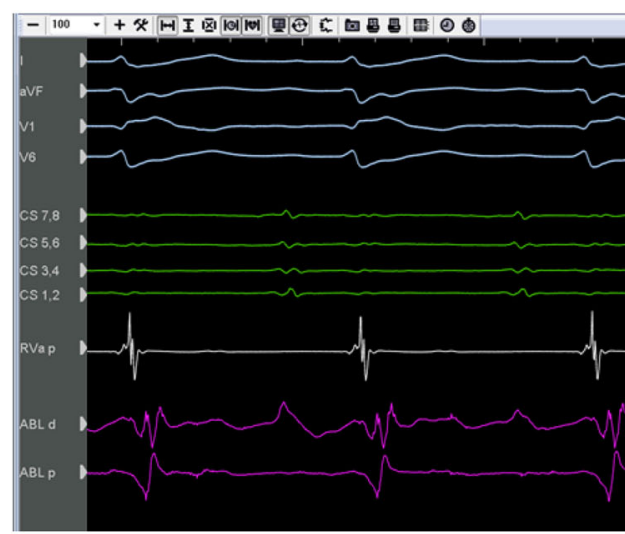

Before ablation

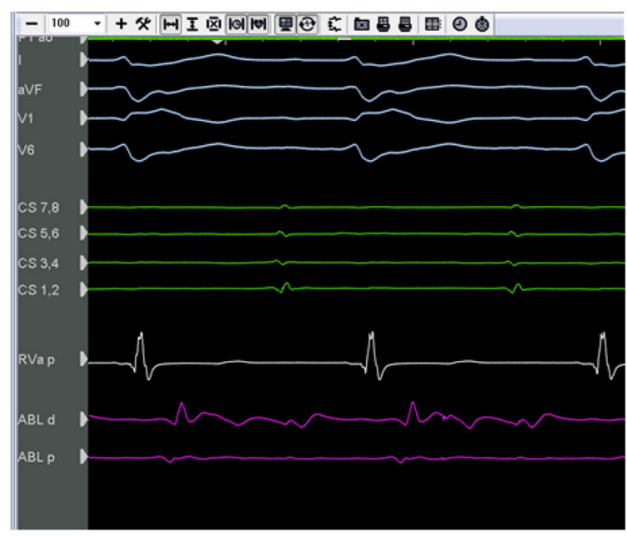

Before ablation

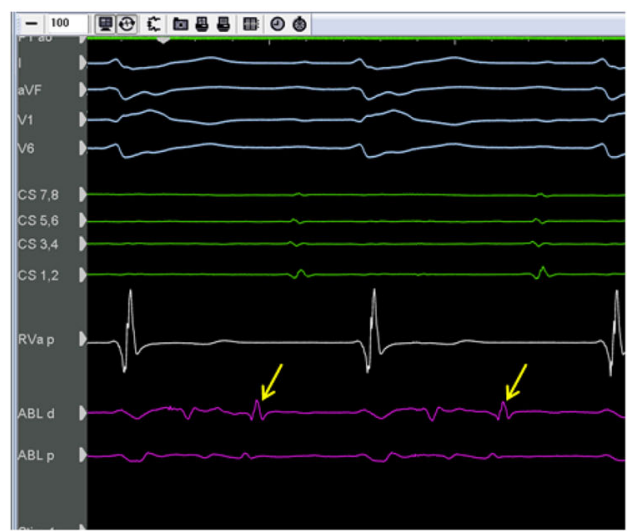

Before ablation

Fig. 4 Bipolar electrograms showing late fractionated potentials in the right ventricular outflow tract (RVOT) endocardium (panel a), late fractionated potentials in the RVOT epicardium (panel b), and isolated diastolic

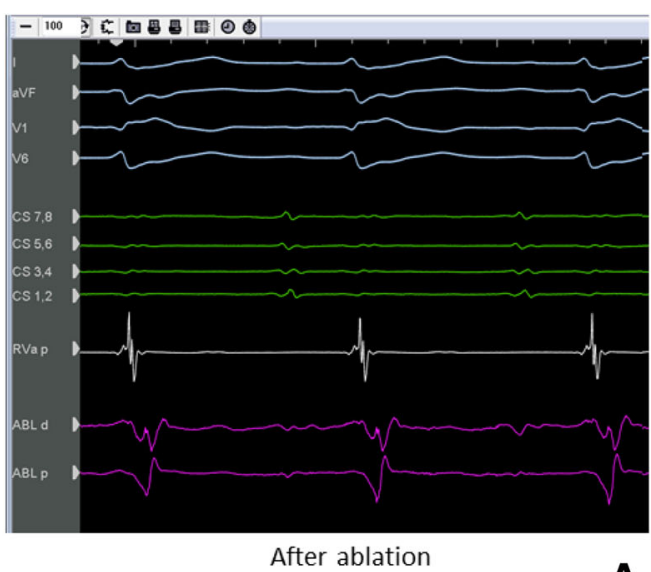

A

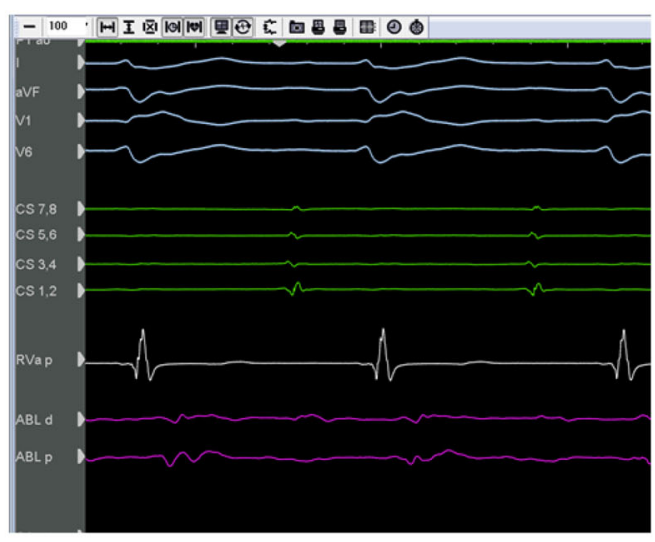

After ablation

B

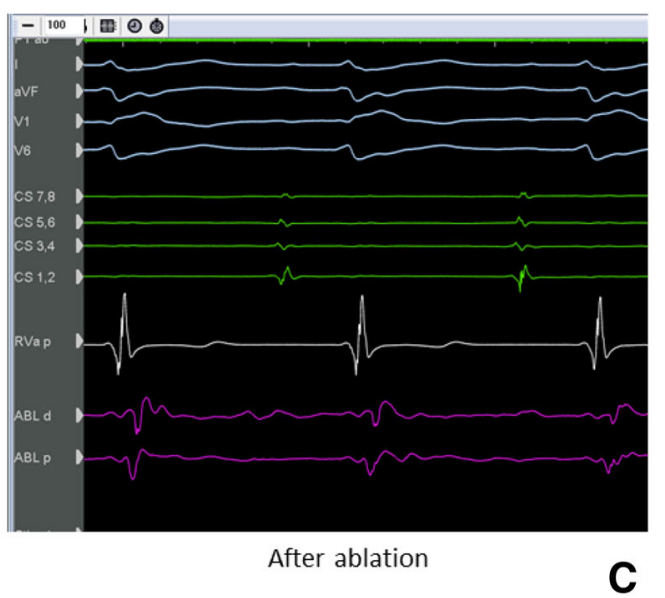

potentials (yellow arrows) in the RVOT epicardium (panel c) which were targeted for ablation. In each panel, the electrograms on the left are before ablation, while the electrograms on the right are after ablation 

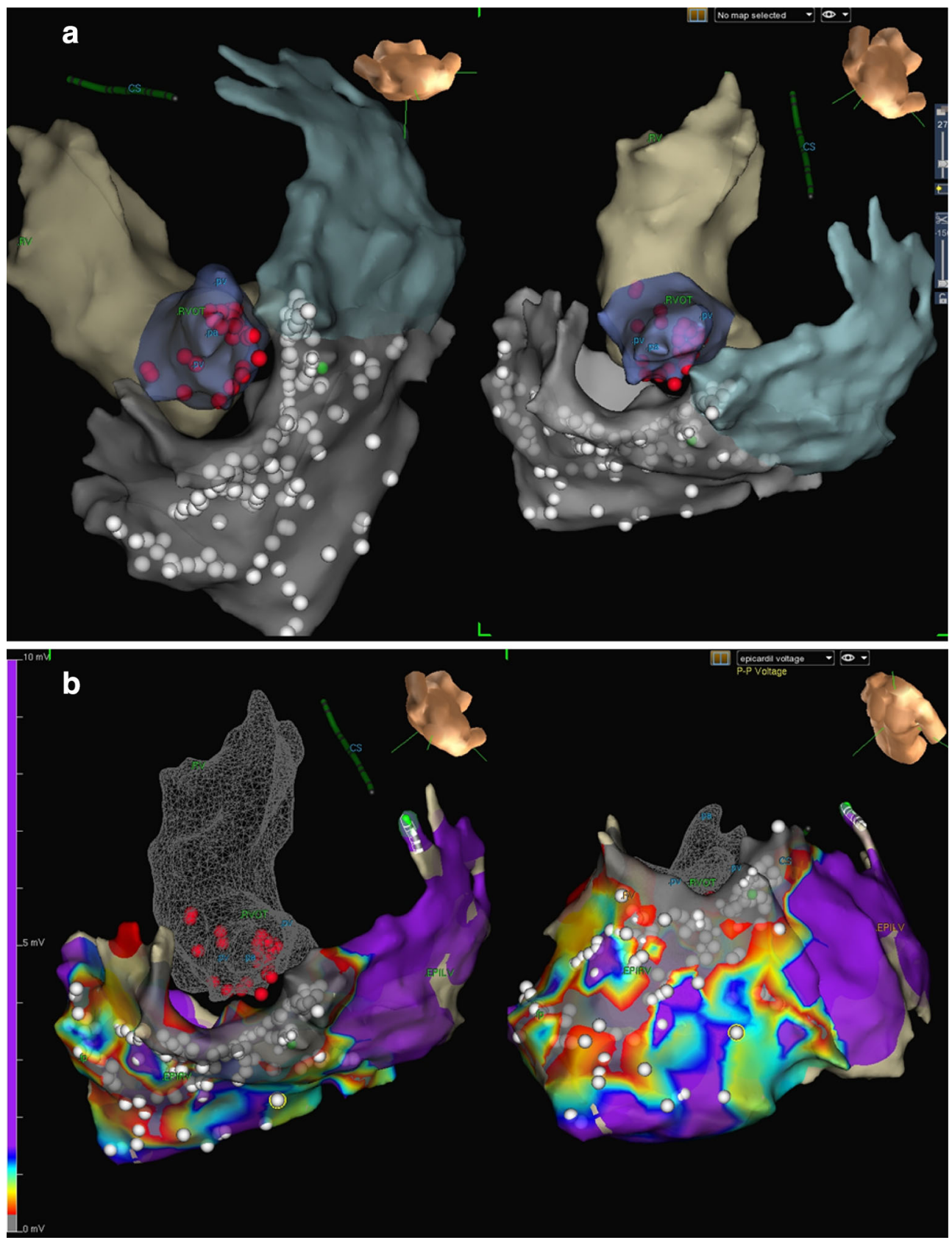

Fig. 5 a 3-D map of the endocardial surface of the right ventricular outflow tract (RVOT) and proximal pulmonary artery (deep blue) and epicardial surfaces of the RVOT and RVOT (gray). Radiofrequency ablation using an irrigated tip catheter was performed endocardially (red dots) and epicardially (white dots). b 3-D ablation map

dysfunction due to changes in transmembrane potential [14], and have pro-arrhythmic effects [15]. Medications such as quinidine may be (white dots) superimposed on the electroanatomic voltage map of the right ventricular outflow tract (RVOT) and anterior RV wall. Areas with low-voltage, late, fractionated electrograms were targeted for ablation. The endocardial ablation map (red dots) in the RVOT is also shown for reference purposes

effective in reducing ventricular arrhythmias in BrS patients, but adverse effects and drug-drug interactions may preclude their long-term use 
[3]. This is especially true for younger patients, who may need these medications for decades.

Reduction in the inward sodium current $\left(\mathrm{I}_{\mathrm{Na}}\right)$ in $\mathrm{BrS}$ patients accentuates the action potential (AP) phase 1 notch caused by the transient outward potassium current $\left(\mathrm{I}_{\mathrm{to}}\right)$, resulting in loss of the dome pattern of phase 2 (inhibition of inward calcium current) of the cardiac AP and shortening of the AP duration (Fig. 6) [16]. Regions with higher $\mathrm{I}_{\text {to }}$ density (such as the RVOT epicardium) are most susceptible to the loss-of-function $\mathrm{I}_{\mathrm{Na}}$ channel mutations present in the majority of BrS patients. Studies from canine cardiac preparations suggest that $I_{\text {to }}$ currents are more prominent in male hearts compared to females, which may explain the higher prevalence of the BrS phenotype in males [17]. In addition, differential effects of the reduction in $\mathrm{I}_{\mathrm{Na}}$ in the RVOT epicardium and endocardium result in a voltage gradient between the RVOT endocardium and epicardium. $\mathrm{I}_{\text {to }}$ density is also higher in some RVOT epicardial sites than others. These perturbations lead to marked transmural and epicardial dispersion of repolarization, which, combined with the conduction delay caused by reduced $\mathrm{I}_{\mathrm{Na}}$, leads to phase 2 reentry. These phase 2 reentry circuits lead to development of PVCs with short coupling intervals, which can be triggers for VT/VF in BrS patients $[9,18]$ (Figs. 7, 8).

Reduced $\mathrm{I}_{\mathrm{Na}}$ in $\mathrm{BrS}$ patients may also manifest a prolonged PR interval, as well as a prolonged $\mathrm{HV}$ interval and increased QRS

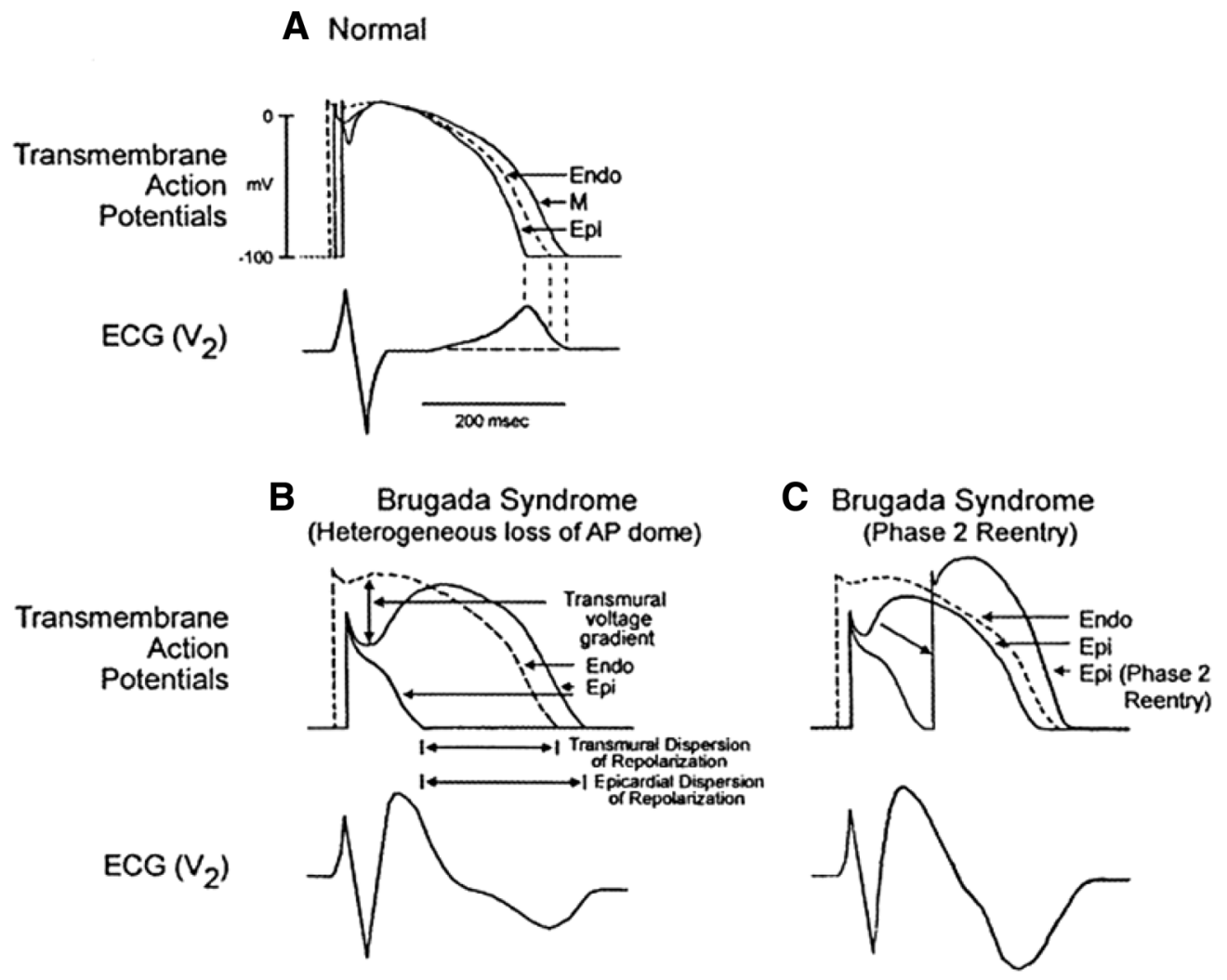

Fig. 6 Loss of action potential dome results in a transmural voltage gradient and sets the stage for phase 2

College of Cardiology volume 39, issue 12, Antzelevitch reentry (Reprinted from the Journal of the American 
A
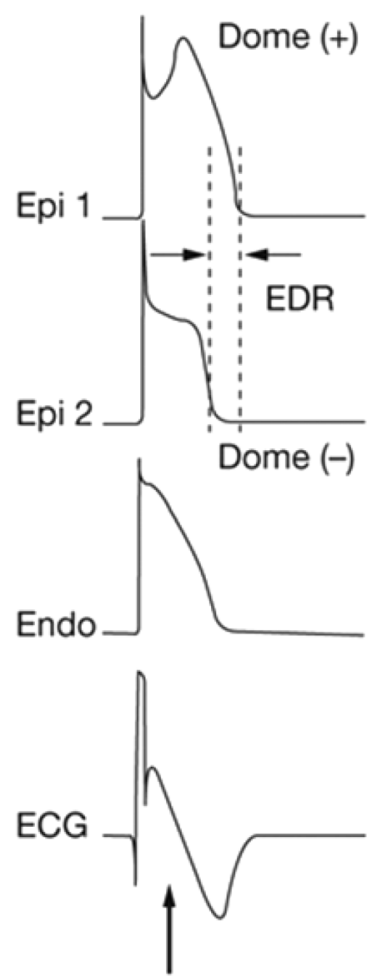

Coved-type ST elevation
B
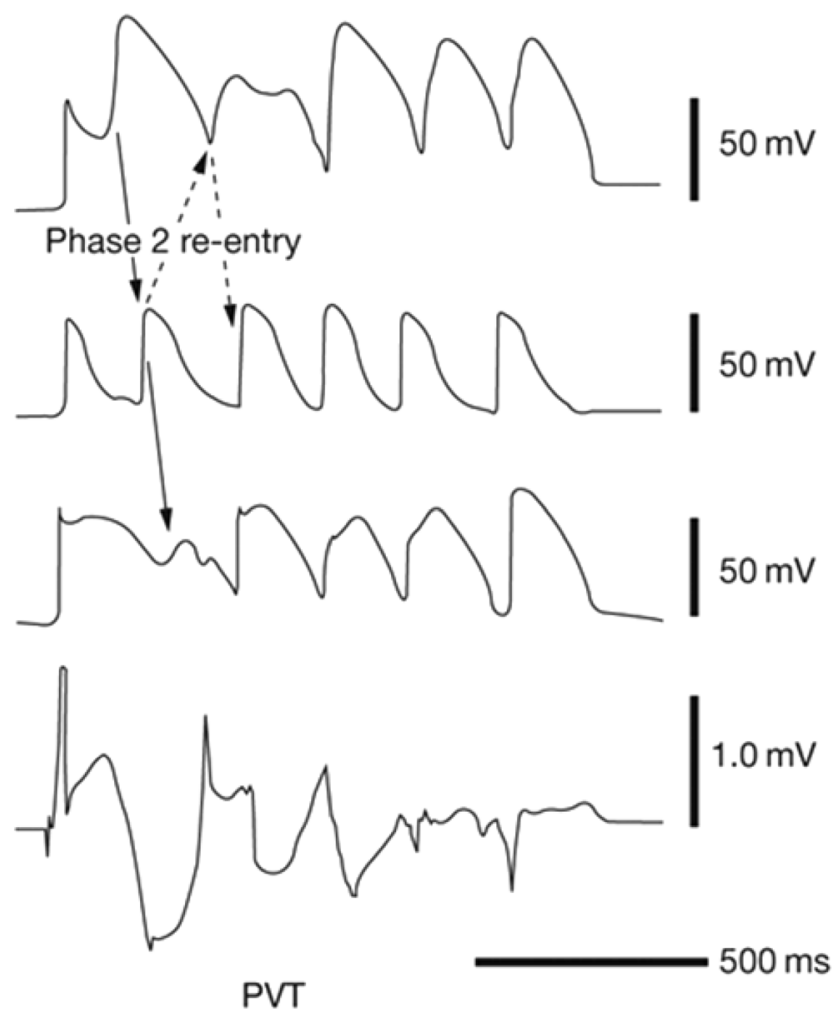

model of Brugada syndrome Reprinted by permission from Macmillan Publishers Ltd: Nature Reviews Cardiology,

Shimizu et al. [18]
Fig. 7 Coved-type ST-segment elevation and subsequent non-sustained polymorphic ventricular tachycardia caused by premature beats induced by phase 2 reentry in a canine

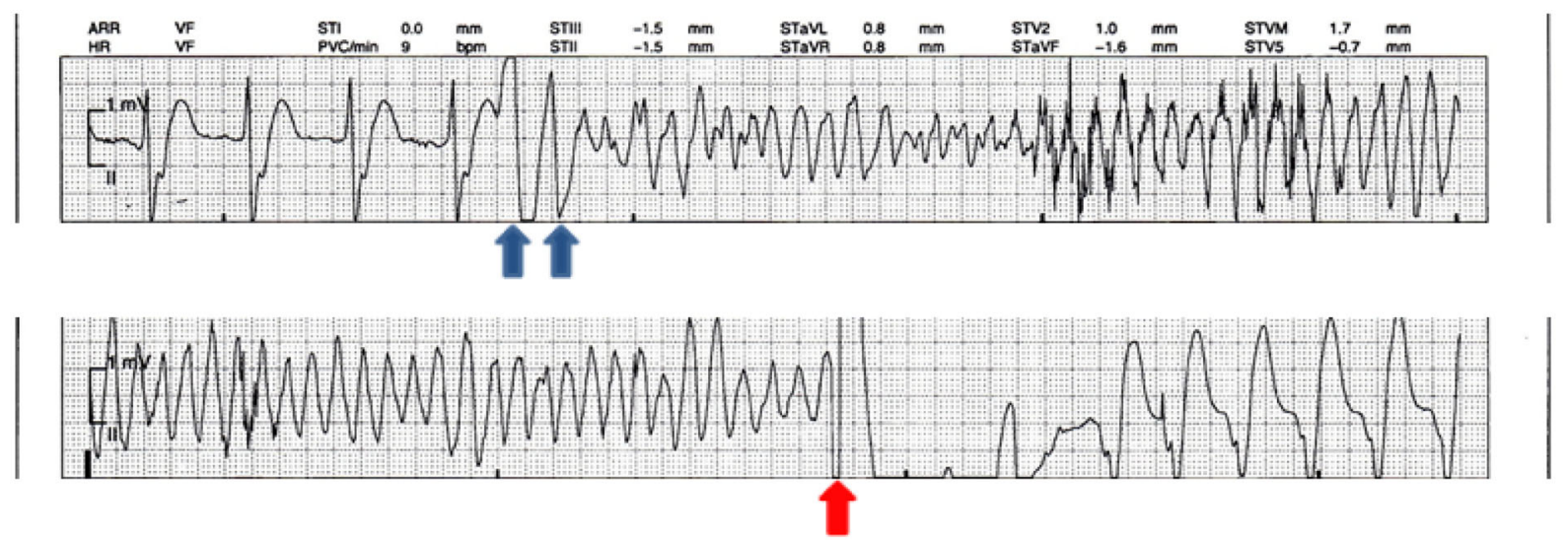

Fig. 8 Surface telemetry strips showing coupled premature ventricular complexes (blue arrows) as the initiating event prior to the onset of ventricular fibrillation (top panel) and conversion to normal sinus rhythm after a shock (red arrow) from the implantable cardioverter-defibrillator 
duration (due to slowing of His-Purkinje and ventricular conduction). Reduction in intraventricular conduction velocity causes development of low-voltage, high-frequency signals that occur after the QRS complex on intracardiac electrograms, which are more pronounced in the RVOT epicardium in BrS patients. These high-frequency late potentials may be due to localized, concealed phase 2 reentry, and the low-voltage fractionated signals are thought to correspond to the second AP upstroke after the accentuated notch, which occurs heterogeneously within the RVOT epicardium. Such delayed and fractionated electrogram signals have also been demonstrated on endocardial mapping of the $\mathrm{RV}[19,20]$. Late potentials as seen on signal-averaged ECG were shown to be an independent predictor of arrhythmic events in a small study of BrS patients [21], but larger studies exploring this association are lacking.

Ablative techniques for management of $\mathrm{BrS}$ patients initially targeted the short coupled PVCs in the RVOT, which may be triggers for the development of VT/VF episodes. Haissaguerre and colleagues [5] reported a series of seven patients (three of whom had $\mathrm{BrS}$ ) with documented polymorphic VT or VF, who underwent an EP study and mapping to detect focal ectopic ventricular 'triggers' for the VT/VF episodes. Mapping revealed that the earliest electrogram relative to the onset of the ectopic QRS complex was located within the RVOT (two patients) or the right-sided Purkinje system (one patient). Endocardial ablation of these sites resulted in non-inducibility of VT/VF post-ablation, and was associated with reduced recurrence of VT/VF episodes during short-term (mean $7 \pm 6$ months) follow-up. Isolated reports using this endocardial approach followed in the literature [22]. Unfortunately, a significant number of BrS patients undergoing EP study may not demonstrate focal PVCs at baseline, even with aggressive stimulation protocols, thereby limiting the usefulness of this approach [7, 23].

Recent studies have suggested that epicardial substrate modification may be effective in preventing ventricular arrhythmias in BrS patients. In a series of nine symptomatic patients with type 1 Brugada pattern and multiple VF episodes, Nademanee and colleagues showed that epicardial mapping revealed a unique pattern of abnormal, low-voltage, fractionated potentials that occurred late (50-100 ms after the end of the surface QRS complex) which were localized to the anterior aspect of the RVOT. RF ablation of these potentials led to non-inducibility of VT/VF episodes, normalized the Brugada pattern on surface ECG, and prevented VT/VF recurrence in the majority of patients. These authors hypothesized that these areas of slow conduction represent areas of delayed depolarization due to micro-anatomic or functional derangements in conduction between cardiomyocytes, which lead to the characteristic ST-segment elevation pattern seen in BrS [7]. This study led to case reports of epicardial ablation for prevention of ventricular arrhythmias in $\mathrm{BrS}$ patients refusing ICD implantation [24] and recurrent VT/VF [25]. Recently, Brugada, Pappone and colleagues also reported that ablation of low-voltage areas on the epicardial surface of the anterior RVOT and anterior RV free wall was associated with non-inducibility of VT/VF in the electrophysiology lab, and normalization of the surface ECG in $14 \mathrm{BrS}$ patients who had received an ICD [8].

The use of ablation (whether epicardial or endocardial) for the management of Brugada storm has rarely been reported. Nakagawa and 
colleagues reported a case of a forty-one-year-old man with Brugada storm who was found to have a PVC originating from the posterolateral wall of the RVOT that consistently initiated his VF episodes, and endocardial RF ablation of this PVC eliminated his VF episodes, in spite of the co-existence of other PVC morphologies in the RVOT and RV inflow tract [26]. In a series of four patients with a history of VF storm, Sunsaneewitayakul and colleagues showed that endocardial RF ablation of late activated zones in the RVOT seen on non-contact mapping was associated with normalization of the Brugada pattern on surface ECG, however, recurrent episodes of VF (including VF storm) were recorded in three of the four patients post-ablation [23]. Cortez-Dias and colleagues performed epicardial ablation in a 60-year-old woman with $\mathrm{BrS}$ and frequent episodes of polymorphic VT/VF refractory to quinidine. They targeted bipolar electrograms with delayed fractionated potentials (up to $370 \mathrm{~ms}$ after the surface QRS complex) on the anterior RVOT wall. The surface ECG normalized six weeks after ablation, and no recurrent VT/ VF episodes were observed over a 6-month follow-up [25].

A combined (epicardial-endocardial) ablation strategy for Brugada storm, targeting low-voltage, fractionated, late potentials in the RVOT in the absence of spontaneous or induced PVC 'triggers' on an EP study, has not been previously reported in the literature. In addition, we report the longest follow-up duration (forty-one months) for a patient undergoing an ablation-based strategy for management of $\mathrm{BrS}$ in the published literature. We were unable to pursue a focal ablation strategy due to the absence of spontaneous or induced PVCs in the EP lab, which (as noted) is not an uncommon scenario in BrS patients. In the context of electrical storm in a young patient with BrS who had already demonstrated intolerance to quinidine, we employed a combined ablation strategy consisting of ablation of both epicardial and endocardial late potentials within the RVOT, to eradicate areas of delayed depolarization and thereby reduce or eliminate the substrate conducive to sustained episodes of VT/VF. We propose that the absence of PVC 'triggers' on EP study should prompt the operator to look for late, low-voltage, fractionated potentials in the RVOT, and target these sites identified with electroanatomic mapping for ablation. Although the Brugada pattern on surface ECG persists in this patient even after forty-one months of follow-up (Fig. 9), he has had no recurrences of VT/VF since the ablation procedure. He remains on cilostazol after the ablation as per the referring clinician's preference.

There is conflicting data in the literature regarding the utility of cilostazol for the prevention of ventricular arrhythmias in BrS patients [27, 28]. Current guidelines for management of $\mathrm{BrS}$ do not include cilostazol as a potential therapy for patients with $\mathrm{BrS}$ [2]. Other experts currently give a class IIb recommendation for the use of cilostazol for arrhythmia suppression in BrS patients [29]. Our patient continued to have persistent palpitations during the short duration (two months) of therapy with cilostazol prior to the ablation. Therefore, we believe it is unlikely that this medication alone has provided such sustained control of his ventricular arrhythmias.

The development of hyperkalemia, metabolic acidosis, acute kidney injury, and rhabdomyolysis in this patient was attributed to the use of sevoflurane and ephedrine during the ablation procedure. Isolated cases of severe 


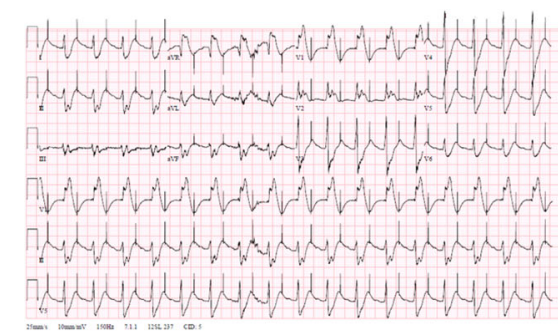

A

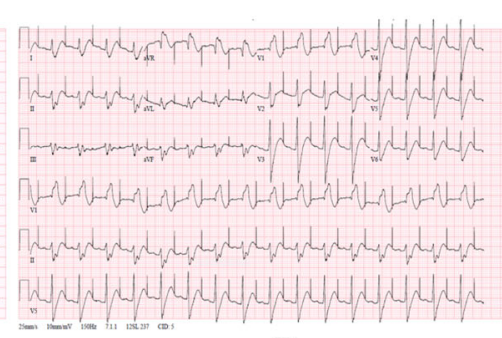

B

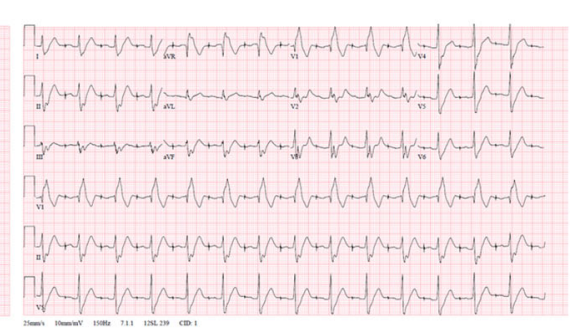

C

Fig. 9 Surface electrocardiograms (ECG) of our patient. Panel a pre-ablation ECG. Panel b post-ablation ECG. Panel c follow-up ECG after 41 months of follow-up

hyperkalemia and rhabdomyolysis in the peri-operative period have been reported with the use of inhaled anesthetics such as sevoflurane [30]. It is plausible that the concomitant use of sevoflurane and ephedrine may have potentiated this risk. Aggressive correction of hyperkalemia and associated cardiac arrhythmias is recommended in these situations, followed by subsequent testing for latent neuromuscular disease. Our patient was offered genetic testing as well as muscle biopsy testing after this event, but he declined.

Combined epicardial-endocardial ablation techniques have been used to treat ventricular tachyarrhythmias in other clinical situations. Bai and colleagues reported a case series of forty-nine patients with arrhythmogenic right ventricular dysplasia/cardiomyopathy (ARVD/ C) and recurrent VTs or multiple ICD therapies in spite of anti-arrhythmic medications, who underwent either an endocardial-only VT ablation $(n=23)$ or a combined endo-epicardial VT ablation $(n=26)$ in a non-randomized fashion. After follow-up for at least three years, patients in the combined endo-epicardial group had significantly more freedom from ICD therapies or ventricular arrhythmias compared to the endocardial-only group (84.6\% versus 52.2\%, $p=0.029)$ [31]. Recent research suggests that there is considerable overlap in clinical phenotype as well as the molecular mechanisms responsible for ventricular tachyarrhythmias among patients with BrS and ARVD/C [32]. Combined endo-epicardial ablation has also been shown, in small studies, to be feasible and safe for treating monomorphic VT refractory to anti-arrhythmic medications in selected patients with hypertrophic cardiomyopathy [33].

\section{CONCLUSION}

We describe the first reported case of combined (epicardial and endocardial) ablation of abnormal, low-voltage, fractionated late potentials within the RVOT (in the absence of spontaneous or induced ventricular ectopic beats on EP study) for the treatment of Brugada storm. Post-ablation, our patient has not had any recurrences of VT/VF over a follow-up period of forty- one months, the longest follow-up duration for any BrS patient treated with ablation reported in the literature. Although he remains on pharmacotherapy with oral cilostazol following the procedure, it is unlikely that cilostazol alone produced such sustained control of his ventricular arrhythmia. We propose that a combined ablation of late potentials in the RVOT may be a useful approach for management of selected patients with BrS. 


\section{ACKNOWLEDGMENTS}

No funding or sponsorship was received for this study or publication of this article. All named authors meet the International Committee of Medical Journal Editors (ICMJE) criteria for authorship for this manuscript, take responsibility for the integrity of the work as a whole, and have given final approval for the version to be published.

Disclosures. Kousik Krishnan reports receiving fellowship funding support from St. Jude Medical, Inc., Boston Scientific Corporation, and Medtronic, Inc.; and being a member of the speaker's bureau for Zoll Medical Corporation. Richard G. Trohman reports serving or having served as an advisor to Boston Scientific Corporation/Guidant; receiving research grants from Boston Scientific Corporation/Guidant, Medtronic, Inc., St Jude Medical, Inc., Vitatron, and Wyeth-Ayerst/Wyeth Pharmaceuticals; serving or having served as a consultant for Biosense Webster Inc. and St Jude Medical, Inc.; and receiving speakers fees or honoraria from Boston Scientific Corporation/Guidant CRM, Medtronic, Inc, Daiichi-Sankyo and St Jude Medical, Inc. Christopher Madias and Sandeep A. Saha have nothing to disclose.

\section{Compliance with ethics} guidelines. Informed consent was obtained from the patient for being included in this report.

Open Access. This article is distributed under the terms of the Creative Commons AttributionNonCommercial 4.0 International License (http://creativecommons.org/licenses/by-nc/4. $0 /$ ), which permits any noncommercial use, distribution, and reproduction in any medium, provided you give appropriate credit to the original author(s) and the source, provide a link to the Creative Commons license, and indicate if changes were made.

\section{REFERENCES}

1. Brugada J, Brugada R, Brugada P. Right bundle-branch block and ST-segment elevation in leads V1 through V3: a marker for sudden death in patients without demonstrable structural heart disease. Circulation. 1998;97(5):457-60.

2. Priori SG, Wilde AA, Horie M, Cho Y, Behr ER, Berul $\mathrm{C}$, et al. Executive summary: HRS/EHRA/APHRS expert consensus statement on the diagnosis and management of patients with inherited primary arrhythmia syndromes. Europace. 2013;15(10):1389-406.

3. Belhassen B, Rahkovich M, Michowitz Y, Glick A, Viskin S. Management of Brugada syndrome: thirty-three-year experience using electrophysiologically guided therapy with class 1A antiarrhythmic drugs. Circ Arrhythm Electrophysiol. 2015;8(6):1393-402.

4. Ohgo T, Okamura H, Noda T, Satomi K, Suyama K, Kurita T, et al. Acute and chronic management in patients with Brugada syndrome associated with electrical storm of ventricular fibrillation. Heart Rhythm. 2007;4(6):695-700.

5. Haissaguerre $M$, Extramiana $F$, Hocini $M$, Cauchemez B, Jais P, Cabrera JA, et al. Mapping and ablation of ventricular fibrillation associated with long-QT and Brugada syndromes. Circulation. 2003;108(8):925-8.

6. Morita H, Zipes DP, Morita ST, Lopshire JC, Wu J. Epicardial ablation eliminates ventricular arrhythmias in an experimental model of Brugada syndrome. Heart Rhythm. 2009;6(5):665-71.

7. Nademanee K, Veerakul G, Chandanamattha $P$, Chaothawee L, Ariyachaipanich A, Jirasirirojanakorn $\mathrm{K}$, et al. Prevention of ventricular fibrillation episodes in Brugada syndrome by catheter ablation over the anterior right ventricular outflow tract epicardium. Circulation. 2011;123(12):1270-9.

8. Brugada J, Pappone C, Berruezo A, Vicedomini G, Manguso F, Ciconte G, et al. Brugada syndrome phenotype elimination by epicardial substrate ablation. Circ Arrhythm Electrophysiol. 2015;8(6):1373-81. 
9. Antzelevitch C, Patocskai B. Brugada syndrome: clinical, genetic, molecular, cellular, and ionic aspects. Curr Probl Cardiol. 2016;41(1):7-57.

10. Benito B, Sarkozy A, Mont L, Henkens S, Berruezo A, Tamborero D, et al. Gender differences in clinical manifestations of Brugada syndrome. J Am Coll Cardiol. 2008;52(19):1567-73.

11. Ingles J, Sarina $T$, Kasparian N, Semsarian C. Psychological wellbeing and posttraumatic stress associated with implantable cardioverter defibrillator therapy in young adults with genetic heart disease. Int J Cardiol. 2013;168(4):3779-84.

12. Tomzik J, Koltermann KC, Zabel M, Willich SN, Reinhold T. Quality of life in patients with an implantable cardioverter defibrillator: a systematic review. Front Cardiovasc Med. 2015;3(2):34.

13. Avitall B, Port S, Gal R, McKinnie J, Tchou P, Jazayeri $\mathrm{M}$, et al. Automatic implantable cardioverter/defibrillator discharges and acute myocardial injury. Circulation. 1990;81(5):1482-7.

14. Neunlist M, Tung L. Dose-dependent reduction of cardiac transmembrane potential by high-intensity electrical shocks. Am J Physiol. 1997;273(6 Pt 2):H2817-25.

15. Vollmann D, Luthje L, Vonhof S, Unterberg C. Inappropriate therapy and fatal proarrhythmia by an implantable cardioverter-defibrillator. Heart Rhythm. 2005;2(3):307-9.

16. Antzelevitch C. Late potentials and the Brugada syndrome. J Am Coll Cardiol. 2002;39(12):1996-9.

17. Di Diego JM, Cordeiro JM, Goodrow RJ, Fish JM, Zygmunt AC, Perez GJ, et al. Ionic and cellular basis for the predominance of the Brugada syndrome phenotype in males. Circulation. 2002;106(15):2004-11.

18. Shimizu W, Aiba T, Kamakura S. Mechanisms of disease: current understanding and future challenges in Brugada syndrome. Nat Clin Pract Cardiovasc Med. 2005;2(8):408-14.

19. Notarstefano P, Pieroni M, Guida R, Rio T, Oliva A, Grotti S, et al. Progression of electroanatomic substrate and electric storm recurrence in a patient with Brugada syndrome. Circulation. 2015;131(9):838-41.

20. Postema PG, van Dessel PF, de Bakker JM, Dekker LR, Linnenbank AC, Hoogendijk MG, et al. Slow and discontinuous conduction conspire in Brugada syndrome: a right ventricular mapping and stimulation study. Circ Arrhythm Electrophysiol. 2008;1(5):379-86.
21. Ikeda T, Takami M, Sugi K, Mizusawa Y, Sakurada H, Yoshino $H$. Noninvasive risk stratification of subjects with a Brugada-type electrocardiogram and no history of cardiac arrest. Ann Noninvasive Electrocardiol. 2005;10(4):396-403.

22. Shah AJ, Hocini M, Lamaison D, Sacher F, Derval N, Haissaguerre M. Regional substrate ablation abolishes Brugada syndrome. J Cardiovasc Electrophysiol. 2011;22(11):1290-1.

23. Sunsaneewitayakul B, Yao Y, Thamaree S, Zhang S. Endocardial mapping and catheter ablation for ventricular fibrillation prevention in Brugada syndrome. J Cardiovasc Electrophysiol. 2012;23(Suppl 1):S10-6.

24. Szeplaki G, Ozcan EE, Osztheimer I, Tahin T, Merkely B, Geller L. Ablation of the epicardial substrate in the right ventricular outflow tract in a patient with Brugada syndrome refusing implantable cardioverter defibrillator therapy. Can J Cardiol. 2014;30(10):1249.e9-11.

25. Cortez-Dias N, Placido R, Marta L, Bernardes A, Sobral S, Carpinteiro L, et al. Epicardial ablation for prevention of ventricular fibrillation in a patient with Brugada syndrome. Rev Port Cardiol. 2014;33(5):305.e1-7.

26. Nakagawa E, Takagi M, Tatsumi H, Yoshiyama M. Successful radiofrequency catheter ablation for electrical storm of ventricular fibrillation in a patient with Brugada syndrome. Circ J. 2008;72(6):1025-9.

27. Abud A, Bagattin D, Goyeneche R, Becker C. Failure of cilostazol in the prevention of ventricular fibrillation in a patient with Brugada syndrome. J Cardiovasc Electrophysiol. 2006;17(2):210-2.

28. Tsuchiya T, Ashikaga $\mathrm{K}$, Honda $\mathrm{T}$, Arita M. Prevention of ventricular fibrillation by cilostazol, an oral phosphodiesterase inhibitor, in a patient with Brugada syndrome. J Cardiovasc Electrophysiol. 2002;13(7):698-701.

29. No authors listed. Potential antiarrhythmic drugs in Brugada syndrome patients. http://www. brugadadrugs.org/antiarrhythmic/. Accessed 27 Aug 2016.

30. Gurnaney H, Brown A, Litman RS. Malignant hyperthermia and muscular dystrophies. Anesth Analg. 2009;109(4):1043-8.

31. Bai R, Di Biase L, Shivkumar K, Mohanty P, Tung R, Santangeli $\mathrm{P}$, et al. Ablation of ventricular arrhythmias in arrhythmogenic right ventricular dysplasia/cardiomyopathy: arrhythmia-free survival after endo-epicardial substrate based mapping and ablation. Circ Arrhythm Electrophysiol. 2011;4(4):478-85. 
32. Corrado D, Zorzi A, Cerrone M, Rigato I, Mongillo $\mathrm{M}$, Bauce $\mathrm{B}$, et al. Relationship between arrhythmogenic right ventricular cardiomyopathy and Brugada syndrome: new insights from molecular biology and clinical implications. Circ Arrhythm Electrophysiol. 2016;9(4). doi:10.1161/ CIRCEP.115.003631.
33. Dukkipati SR, d'Avila A, Soejima K, Bala R, Inada K, Singh S, et al. Long-term outcomes of combined epicardial and endocardial ablation of monomorphic ventricular tachycardia related to hypertrophic cardiomyopathy. Circ Arrhythm Electrophysiol. 2011;4(2):185-94. 